\title{
Timing is crucial for consequences of migratory connectivity
}

\author{
Silke Bauer, Simeon Lisovski and Steffen Hahn
}

S. Bauer (silke.s.bauer@gmail.com) and S. Hahn, Dept of Bird Migration, Swiss Ornithological Inst., CH-6204 Sempach, Switzerland. SB also at: Dept of Animal Ecology, Netherlands Inst. of Ecology (NIOO-KNAW), PO Box 50, NL-6700 AB Wageningen, the Netherlands. - S. Lisovski, Centre for Integrative Ecology, Deakin University, VIC-3220 Geelong, Australia.

\begin{abstract}
Migratory connectivity can have important consequences for individuals, populations and communities. We argue that most consequences not only depend on which sites are used but importantly also on when these are used and suggest that the timing of migration is characterised by synchrony, phenology, and consistency. We illustrate the importance of these aspects of timing for shaping the consequences of migratory connectivity on individual fitness, population dynamics, gene flow and community dynamics using examples from throughout the animal kingdom.

Exemplarily for one specific process that is shaped by migratory connectivity and the timing of migration - the transmission of parasites and the dynamics of diseases - we underpin our arguments with a dynamic epidemiological network model of a migratory population. Here, we quantitatively demonstrate that variations in migration phenology and synchrony yield disease dynamics that significantly differ from a time-neglecting case.

Extending the original definition of migratory connectivity into a spatio-temporal concept can importantly contribute to understanding the links migratory animals make across the globe and the consequences these may have both for the dynamics of their populations and the communities they visit throughout their journeys.
\end{abstract}

Migratory connectivity quantifies the links migrant animals make across the globe and these can have manifold consequences - from individual fitness, population dynamics, gene flow to transmission of pathogens and $\frac{\omega}{\omega}$ parasites. We show through the use of empirical examples and a conceptual model that these consequences not only depend on which sites are used but importantly also on when these are used. Therefore, we specify three dimensions of migration timing - phenology, synchrony and consistency, which describe the timing of migration 1) relative to development of key resources; 2) relative to the migration of other individuals; and 3) relative to previous migration events. Each of these dimensions can alter the consequences, but typically through different mechanisms.

Billions of animals migrate across the globe every year and it is widely acknowledged that the use of different sites has consequences for migrant fitness and the dynamics of their populations as well as for the communities visited (Webster and Marra 2005, Marra et al. 2010, Bauer and Hoye 2014). Migratory connectivity describes the "geographic linking of individuals and populations between one life cycle stage and another" (Webster et al. 2002 p. 76, Salomonsen 1955). It is a concept that can importantly contribute to answering a range of fundamental and applied questions and therefore, has been enthusiastically embraced by the scientific community. Although predominantly applied in studies of migratory birds, it is equally applicable to migratory animals of other taxa (Godley et al. 2010, Miller et al. 2012).

The importance of migratory connectivity is generally acknowledged; yet, most studies describe the degree of connectivity but surprisingly few quantitatively link it explicitly to its consequences. If we wish to develop it towards an explanatory and predictive concept, we need to gain a better understanding of the mechanisms behind the consequences of migratory connectivity and this requires an explicit consideration of time. The consequences of migratory connectivity broadly include those on individual fitness and population dynamics, gene flow and genetic mixing, and community dynamics and ecosystem function. Although it is widely agreed upon that these consequences result from the use of specific sites, conditions on these sites also change over time and therefore, timing of migration will not only shape the magnitude of these consequences but also their nature.

We suggest that the timing of migration is characterised by three dimensions - synchrony, phenology and consistency (which may also be coined as 'variation' around a 'mean' timing and their 'autocorrelation'). Migration synchrony describes how wide-spread over time individuals of a population migrate (Fig. 1). At one extreme, all individuals migrate at the same time - synchronously (Orell et al. 2007), while at the other, individuals migrate at different times - asynchronously. Specific examples of asyn- 


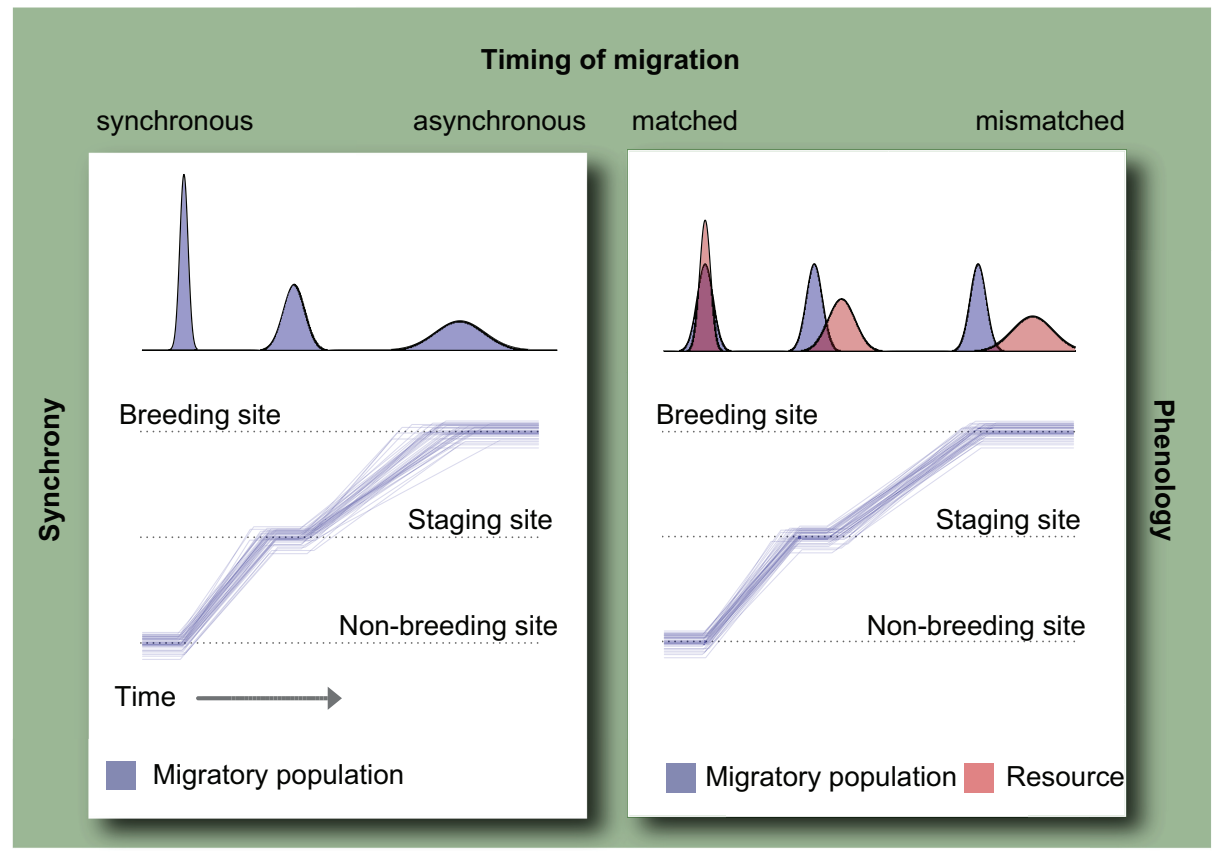

Figure 1. The timing of migration - here exemplarily from a non-breeding site via an intermittent staging to a breeding site - can be characterised by synchrony (left panel) and phenology (right panel). Migration synchrony describes in how far individual migrants depart, stay or arrive at the same time, i.e. synchronously, or at different times, i.e. asynchronously. Migration phenology relates the timing of migration to the phenology of other populations and species, with which migrants interact, e.g. via competition, predation, etc. We exemplarily depicted various degrees of coincidence between migrant visitation and resource availability (upper-right panel) that influence the migrants' fitness and may range from positive under complete overlap to negative when migrant visitation and resource availability are fully mismatched.

chronous migration include differential migration (Colbeck et al. 2013), where (age-, sex- or family-) subgroups of a population migrate at different times. Migration phenology describes the timing of migratory steps - arrival, departure and staging times at sites - relative to the phenology of other relevant processes, e.g. temporal availability of key-resources or presence and abundance of other species and populations (Fig. 1). At the two extremes, the migrants' presence on a particular site fully coincides with, e.g. resource peaks ('matched') or is completely separated from the availability of resources ('mismatched'). Finally, consistency describes how repeatable migration phenology and synchrony are over time - usually over several migrations. (Note that consistency also exists in the spatial sense, i.e. the degree to which migrants return to the same locations in successive migrations (Jorgensen et al. 2010)).

In the following, we illustrate the importance of considering timing for the consequences of migratory connectivity using examples from throughout the animal kingdom, and primarily consider the roles of phenology and synchrony. For a specific example of these consequences - the transmission of pathogens and parasites - we develop a dynamic network model to quantitatively demonstrate how changes in phenology and synchrony can affect disease dynamics. We would like to emphasize that we do not aim at compiling a comprehensive review of the consequences of migratory connectivity here (for such see Boulet and Norris 2006) but hope to stimulate discussion and increase awareness of the effects of variations in migration phenology and synchrony.

\section{Consequences of migratory connectivity shaped by timing}

\section{Individual fitness and population dynamics}

A variety of factors can affect a migrant's fitness (Fig. 2): Abiotic conditions, e.g. temperature, precipitation or wind, influence energy expenditure during residency (e.g. thermoregulation) and locomotion (e.g. flight); resource availability and abundance of competitors determine how fast migrants can replenish fuel reserves (Stahl et al. 2006, Wittwer et al. 2015); and predators pose mortality risks (Middleton et al. 2013) or spark a range of non-lethal effects (Morrissette et al. 2010).

As these factors typically vary between sites, the choice of sites and thus, migratory connectivity can have important implications for individual fitness (Alves et al. 2013) and ultimately, strongly influence a population's demographic rates (Norris and Marra 2007).

Furthermore, all of these factors usually change seasonally but often also at time-scales similar to the visitation of migrants and therefore, their effect may vary even if migrants use the same set of sites. Therefore, variations in the phenology of migration will lead to a population experiencing on average different resource levels, abundances of competitors and predators ("phenological match/mismatch', Johansson et al. 2015), and migration synchrony determines the within-population variation with regard to the overall effects of these factors. If, for instance, resource availability changes as a consequence of natural decay or due to finite resources being exhausted, early migrants 
... determines level of resources available for early and late migrants if resources change over visitation period.

... if conditions change over visitation period, early and late migrants experience different weather conditions with potential fitness consequences.

... determines magnitude of densitydependent interactions.

... determines predation risk, hunting pressure or disturbance levels experienced by early/late migrants if these change over visitation period.

... affects pair formation and mating

... may separate infected and uninfected individuals, changing transmission and prevalence.

... determines whether the input of nutrients or energy results in resource pulses, cascading through the local food web.

... determines whether propagules are inserted simultaneously which enhances probability of establishment, e.g. via mass effect or numerical domination.

... determines interaction strength with resident species and populations.
... relative to phenology of resources determines match or mismatch between migrant requirements and resource availability.

... relative to seasonality of places and variability of weather can affect refuelling and survival.

... relative to phenology of other species sets the specific levels of competition, facilitation or other interactions.

... relative to predator phenology or hunting schedules determines mortality risk experienced by migrants.

... relative to phenology of breeding places can result in variations in reproductive success and contribution to the following generation.

... in relation to variations in susceptibility or environmental parasite pressure can alter prevalence levels over time.

... relative to phenology of community determines the pathways through which inserted nutrients and energy enter local community.

... sets the timing of input relative to the phenology of community, which affects fate and effect of propagules, e.g. probability of establishment, pollination, etc.

... determines which trophic processes in a local community are affected by migrants.

Figure 2. The consequences of migratory connectivity can be manifold, ranging from consequences on individual fitness and population dynamics to those on community dynamics and ecosystem function. All of these consequences can be modified and shaped by the timing of migration - its phenology and synchrony, via a suite of different factors and mechanisms.

would benefit from abundant resources compared to late migrants in an asynchronously migrating population. This is exemplified in a population of Arctic breeding geese, where individuals that arrived at stop-over locations at the peak of vegetation growth had a higher breeding success (Kölzsch et al. 2015).

Similarly, within-population competition may be alleviated under asynchronous migration while it is fully effective under 
synchronous migration (Skoglund et al. 2011), e.g. as in the exclusion of competitively inferior individuals from highquality foraging patches (Eichhorn et al. 2009, Beauchamp 2012). Alternatively, synchronous migration can be beneficial if the joint consumption of a resource increases its quality or productivity, as in the case of grazing by migratory geese on a spring stop-over site (Stahl et al. 2006) or the increased productivity of the African savannah through the temporal grazing of migratory herbivores (Holdo et al. 2007).

The level of predation (incl. hunting) may also change at the time-scale of migrant visitation, e.g. as resulting from seasonal hunting permissions or mobile predators. For instance, hunting on spring-migrating geese in Russia is permitted during 10 days of peak migration and individuals migrating outside this 10-day hunting window experience much lower mortality risks (Mooij et al. 1999). Similarly, late-migrating sandpipers responded to the arrival of predators (peregrine falcons Falco peregrinus) on a common stop-over site with behavioural changes, e.g. increased vigilance, reduced foraging and consequently, reduced migration speed - behaviours that early-migrants failed to show (Hope et al. 2014).

\section{Gene flow and genetic mixing}

Migratory connectivity can influence the degree of gene flow between populations - as a result of either spatial or temporal segregation (Webster and Marra 2005, Bensch et al. 2009, Moussy et al. 2013). Although it is commonly acknowledged that strong (spatial) migratory connectivity can lead to limited or no gene flow, to local adaptations and ultimately, speciation (Bensch et al. 1999, Fraser and Bernatchez 2005), temporal segregation can have the same effects. A prominent example is the European blackcap Sylvia atricapilla, in which there is no or very little gene flow between two sub-populations despite them meeting at a common breeding site. This is mainly explained by differences in arrival and onset of breeding between these sub-populations that segregated them temporally and resulted in assortative mating, restricted gene flow and ultimately, phenotypic divergence (Berthold et al. 1992, Bearhop et al. 2005).

\section{Community dynamics and ecosystem functions}

With their movements, migrants connect widely separated and diverse communities and ecosystems, influence their structure and dynamics through a variety of transport and trophic effects (Bauer and Hoye 2014). Clearly, migratory connectivity describes which communities and ecosystems are linked by migratory movements but the phenology and synchrony of visitation are also profoundly important to assessing the influence migrants can have on these communities.

The timing of migration relative to resident phenology is fundamental to the strength and direction of migrantresident interactions (Yang and Rudolf 2010) and can influence key-features of communities (Nakazawa and Doi 2012). For instance, migrants can only be important pollinators if their visits coincide with peak flowering, e.g. lesser long-nosed bats Leptonycteris yerbabuenae time migration to coincide with peak flowering in the cacti-populations along their way (Fleming 2004). Similarly, if parasite prevalence shows a marked seasonal dynamics, transmission may be restricted to sites where high prevalence and migrant visitation coincide (Hoye et al. 2011).

Effects of migrants on communities can also be shaped by the synchrony of migration. For instance, the simultaneous input of nutrients may constitute resource pulses, which can profoundly alter demographic rates and abundances of interacting populations, with cascading effects that may persist long after the pulse is extinguished (Holt 2008). Also asynchrony in migration can have attendant consequences for communities and ecosystems, e.g. in partially migratory freshwater fish the proportion of the population migrating determines, via various intermediate steps, the transition between alternative stable states in a lake ecosystem (Brodersen et al. 2008).

\section{Transmission of parasites and disease dynamics}

A specific process that highly depends on migratory connectivity and can have implications on several organisational levels is the transmission of parasites and the dynamics of diseases. Infections impair the fitness of migrant hosts, e.g. directly through increased mortality but also indirectly through costly immune responses. Disease symptoms may range from fatigue, reduced foraging or movement (Adelman et al. 2010), which may knock-on to lower fuelling rates, later departure and eventually, in reduced reproductive success or survival. Depending on the proportion of a population being infected and the severity of effects, this may severely influence population demographic rates (Hudson et al. 2002). On the community level, parasites can change the outcome of interactions such as competition and predation and thus, ultimately species coexistence and diversity (Holt and Dobson 2006).

If we want to understand the dynamics of parasites within migratory host populations, we need to consider migratory connectivity and the timing of movements (Altizer et al. 2011, Møller and Szep 2011). If individual migrants visit the same sites at the same times, they are thought to encounter the same variety of parasites and prevalence in the population is driven by local (re-)infections (Hudson et al. 2002). In contrast, if migrants visit different sites or the same sites at different times, they potentially encounter a different diversity and abundance of parasites (Kamiya et al. 2014); once these individuals congregate on a common site, they may harbour, and exchange, a greater variety of parasites (Gaidet et al. 2012).

Considering time explicitly is required for predicting the consequences of migratory connectivity to parasite prevalence and dynamics for several reasons: First, prevalence may vary over time, e.g. resulting from variations in environmental conditions (Reperant et al. 2010), density of potential hosts (Gaidet et al. 2012) or the influx of immunologically naïve individuals, such that there are periods during which transmission is more likely than in others (Hoye et al. 2011). Secondly, infectious individuals need to actually meet susceptible (un-infected) individuals to transmit parasites, which might be efficiently prevented when infected and uninfected individuals migrate at different times. For instance in monarch butterflies Danaus plexippus, individuals infected with a protozoon parasite migrated at lower speeds than their 
healthy conspecifics (Bradley and Altizer 2005) and such "migratory escape" introduced a barrier to the spread of parasites that consequently reduced parasite-prevalence in the population (Altizer et al. 2011, Hall et al. 2014).

To underpin our verbal arguments, we used a simple model to demonstrate how the prevalence of pathogens in a population of migratory hosts may change when migration phenology and synchrony are varied. To this end, we combined a dynamic network model with an epidemiologi$\mathrm{cal}$ and a migration model and followed the prevalence of pathogens in the migratory host population over time (for details see Supplementary material Appendix 1). The population ('network') consisted of individuals ('nodes'), which may be linked via 'edges'. Links between individuals form and dissolve probabilistically over time while the average number of links per individual is preserved. Individuals were additionally characterised by infection status and location and these could change as a result of infection dynamics and migration, respectively.

Infection dynamics. We used a SIS (susceptibleinfectious-susceptible) model (Keeling and Rohani 2008) with no immunity or latent phase. Susceptible individuals can be infected with probability, $\tau$, if connected to an infected individual and infected individuals recover with probability $\gamma$, and re-enter the pool of susceptibles. Although we had no particular pathogen or disease in mind, we used parameter values of $\tau=0.2$ and $\gamma=0.07$, i.e. infections lasted on average 14 days.

Migration. We considered a simple type of migration from a starting location to a destination, i.e. assuming strong migratory connectivity. Migration was instantaneous and at, or around, a mean migration date, $t_{\text {mig }}=100$. As we assumed the two locations to be distant, no links (and thus, no pathogen transmissions) were allowed between individuals at disparate locations.

Scenarios. We changed migration synchrony by varying the standard deviation around the mean migration date from $\sigma=0$ to 10 and thus, from completely synchronous to asynchronous. Please note that the scenario on 'complete synchrony' is equivalent to neglecting any spread in migration over time. Secondly, we introduced a 20-day period of elevated (environmental) pathogen pressure (or increased susceptibility to infections) at one location and varied its onset relative to the timing of migration from day $t=60,90$, to 120 , i.e. before, coinciding with, or after migration, respectively. During this period individuals at the starting location were additionally infected with a probability of 0.5 .

Results. Both migration synchrony and phenology importantly shaped (local) disease dynamics (Fig. 3). Changing synchrony changed the prevalence of pathogens (Fig. 3a-b) such that in a completely synchronously migrating population, prevalence remained at the same level on both starting and destination location. However, if migration was spread out over time, prevalence gradually decreased at the starting location and gradually increased at the destination and was thus considerably lower than under complete synchrony.

Changing the timing of elevated pathogen pressure relative to migration altered pathogen dynamics substantially
(Fig. 3c-d). While pathogen pressure at the starting location obviously could not change prevalence if it was elevated after migration, it significantly increased prevalence when it was elevated before or during migration. The prevalence at departure from the starting location then spilled over to, and influenced, the prevalence at the destination location (Fig. 3d).

Thus, although the underlying epidemiology remained unaltered, both migration synchrony and phenology importantly shaped (local) disease dynamics with the resulting prevalence varying widely and substantially differing from the time-neglecting case. Naturally, our modelling exercise is only a first step and comprehensive studies are required that apply the model to specific migrants, pathogens and parasites, extend it to accommodate more complex migrations of a variety of migrants, and include effects of parasites on host state, behaviour and demographic parameters.

\section{Conclusions}

Migratory connectivity is an important concept for the links migrants make between different parts of the world; its implications are far-reaching and can be immense: the dynamics, conservation and management of migratory populations, the effects of potential habitat and climatic changes (Bauer et al. 2008), structure and dynamics of separated communities (Bauer and Hoye 2014), and the spread of parasites, including those with zoonotic potential (Altizer et al. 2011). In addition to the exclusive consideration of spatial links in its original definition, we have shown here that all potential consequences of migratory connectivity can depend on the timing of migration - its phenology and synchrony.

Migration phenology has long been acknowledged as vital for our understanding of the migrants' population dynamics - numerous studies have shown its importance for individual fitness, population demographic rates as well as for the transmission of parasites and the interactions with resident communities. An individual's phenology results from cues that trigger migration (Bauer et al. 2011, McNamara et al. 2011) and other life-history processes and is the result of adaptations to (local) conditions, to the variability in these conditions, and to interactions within populations or with other species (Reed et al. 2010). The level of synchrony in timing of migration then results from the variability between individuals in the use of and response to these cues and the conditions experienced (Harrison et al. 2011). Additionally, migration synchrony might be influenced by a variety of processes, e.g. variation in fuelling rates (Seewagen et al. 2013), sex-specific constraints and selection pressures (Saino et al. 2010), or delayed departure of infected individuals (Hoye et al. 2012), and it may vary for different migratory steps or between breeding and non-breeding migration. Furthermore, the level of migration synchrony will be generally higher in migrants travelling in groups, e.g. fish shoals, herds, swarms, as migration routes and timing result from group decisions (Conradt and List 2009) and with a cultural transmission of migration behaviour (Harrison et al. 2010).

One might argue that the original definition of migratory connectivity implicitly contains a time-dimension as migrants visit the various places at different times of the 
(a)

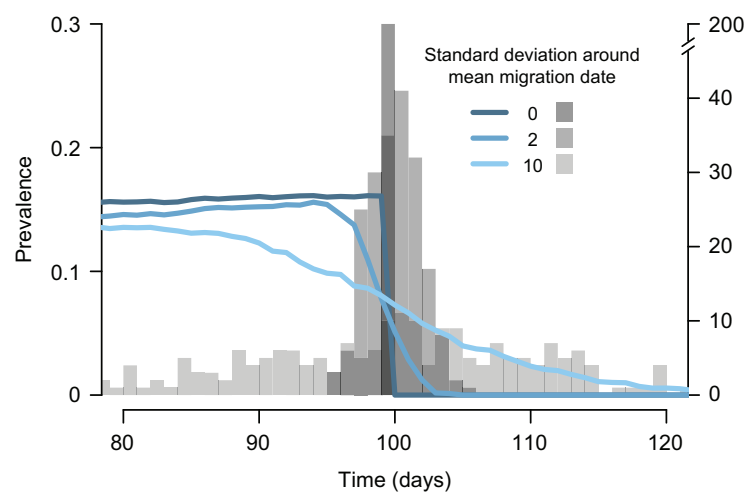

(b)

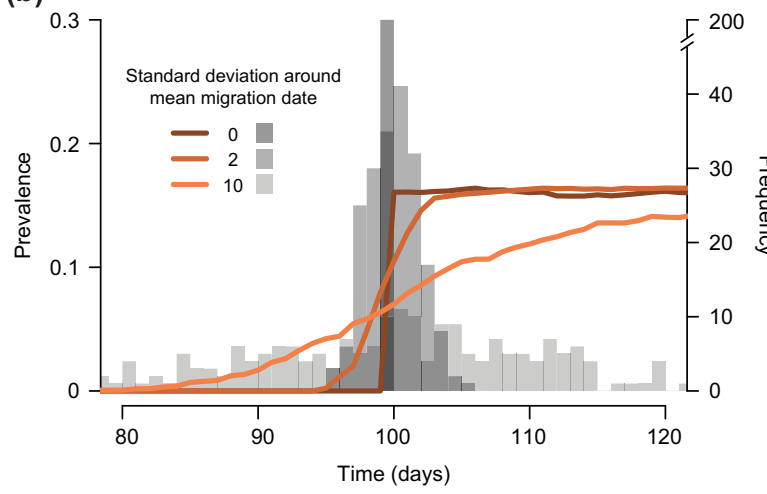

(c)

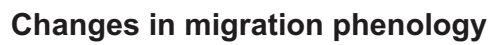

(d)

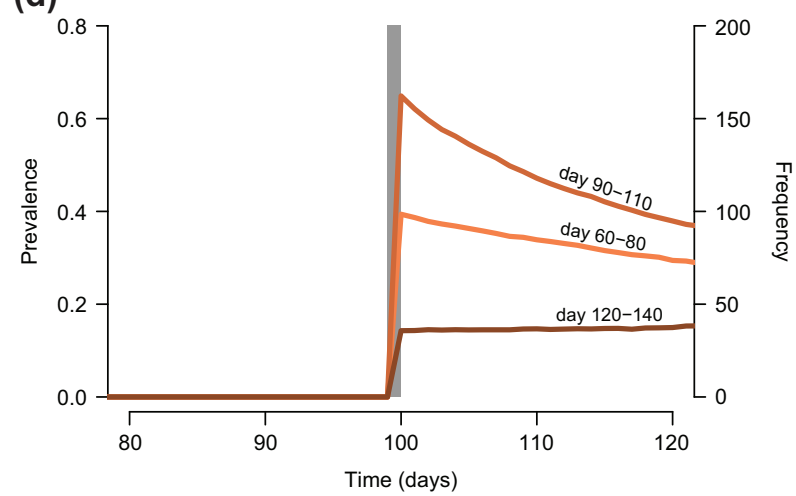

Figure 3. Disease dynamics differed considerably in a dynamic network. Prevalence varied between the two locations (a-c starting location, $\mathrm{b}-\mathrm{d}$ destination) and in dependence of migration synchrony $(\mathrm{a}-\mathrm{b})$ and phenology $(\mathrm{c}-\mathrm{d})$. Increasing variation around the mean migration date makes migration more asynchronous, which is exemplarily illustrated by underlying histograms: completely synchronous (dark grey bars), and increasingly more asynchronous (medium and light grey bars). While in the highly synchronized migration (dark blue and dark orange lines) prevalence was similar at the two locations, asynchronous migration led to slowly decreasing prevalence in the starting location (a) and a slowly increasing prevalence at the destination (b). Prevalence was also affected by the onset of a period of increased parasite pressure, which was varied relative to migration date $(\mathrm{c}-\mathrm{d})$. The highest prevalence resulted when migration coincided with the period of increased parasite pressure, while it was considerably lower for an onset before, and unchanged for an onset after, migration.

year. However, we think that time needs to be made explicit, often at a higher resolution than implicitly contained in the original definition, as we would otherwise neglect consequences that are directly shaped by phenology and synchrony of migration. [An analogy of the implicit-versus-explicit consideration of time could be drawn from network analyses: In most ecological applications to date, networks are considered as time-aggregated networks; however, the dynamics, resilience and stability of time-ordered networks can be fundamentally different from time-aggregated networks (Blonder et al. 2012).]

As we have exemplarily shown, the dynamics of pathogen infections in migratory populations can substantially differ if the timing of migration is considered explicitly - changing synchrony and phenology altered population prevalence over long periods although the underlying infection dynamics remained unchanged. Neglecting that migrations can be spread over time - as in the original definition of migratory connectivity - implicitly assumes that migrations are completely synchronous and disregards the relevance of other processes, which, however, are crucial for understanding its consequences.
Obviously, the relevance of timing may vary, e.g. between periods and places, but whether timing can be neglected or not depends on the consequences of migratory connectivity under consideration and their hypothesized mechanisms. Both consequences and their underlying mechanisms determine which spatial and temporal scales are required for their detection, and in turn, the spatial and temporal scales required have implications for the choice of empirical methods: Various tracking methods exists to date, all of which set very different yet strict limits to the spatial and temporal resolutions that can be achieved (Boulet and Norris 2006), and therefore, the choice of methodology determines, and possibly restricts, the consequences and mechanisms that can be identified.

This sets the basis for several exciting challenges for future research. A prime need is a sound theoretical basis for the consequences of migratory connectivity and the role of timing in shaping these. In particular, we need theoretical studies that develop predictions for specific consequences of migratory connectivity and explore their mechanisms (Taylor and Norris 2010), investigating the respective roles of phenology, synchrony and consistency in influencing the 
fitness of individuals, population dynamics, gene flow as well as community dynamics and ecosystem functioning. Network approaches might be particularly useful when consequences are modified by heterogeneous contact structures that change over time. For instance, several community and ecosystem consequences of migratory connectivity result from direct interactions ('contacts') between migrants and residents but the intensity and nature of these interactions critically depend on the numbers of migrants and how these may vary over time - their phenology and synchrony (Fig. 2). Whether parasites will be successfully introduced into a resident community, which pathways imported nutrients and energy take, or whether dispersed seeds establish in a resident community - depend on phenology and synchrony of migration and can be explored with dynamic network models.

A complimentary approach are behaviour-based migration models, which can explicitly take into account behavioural flexibility and constraints in responding to climatic and habitat changes (Hedenström et al. 2007, Barta et al. 2008, Fagan et al. 2012), the variable number and importance of sites that constitute migration routes (Iwamura et al. 2013) but also fundamental differences in migration strategies, such as different modes of locomotion (Hein et al. 2012)

Although for many species and populations we are still at the stage of identifying the places to which individuals migrate, we urge for an extension of the original, exclusively spatial definition of migratory connectivity into a spatiotemporal concept. Going beyond the descriptive stage of migratory connectivity requires us to be explicit about its consequences, their mechanisms and the spatial and temporal scales alike.

Acknowledgements - All authors conceived and discussed the conceptual framework and wrote the manuscript. SB developed and analysed the epidemiological network model. We thank B. J. Hoye, L. Jenni, B. Nolet, W. A. Buttemer and J. Gill for valuable comments on earlier versions of this manuscript. Part of the work has been accomplished while SB and SH were on research leave at the Centre for Integrative Ecology, Deakin Univ. Geelong, Australia. This is part of a Swiss National Science Foundation project (31003A_160265) and publication 5955 of the Netherlands Institute of Ecology (NIOO-KNAW). The authors declare no conflict of interest.

\section{References}

Adelman, J. S. et al. 2010. Radiotelemetry reveals variation in fever and sickness behaviours with latitude in a free-living passerine. - Funct. Ecol. 24: 813-823.

Altizer, S. et al. 2011. Animal migration and infectious disease risk. - Science 331: 296-302.

Alves, J. A. et al. 2013. Costs, benefits, and fitness consequences of different migratory strategies. - Ecology 94: 11-17.

Barta, Z. et al. 2008. Optimal moult strategies in migratory birds. - Phil Trans. R. Soc. B 363: 211-229.

Bauer, S. and Hoye, B. J. 2014. Migratory animals couple biodiversity and ecosystem functioning worldwide. - Science 344: 1242552.

Bauer, S. et al. 2008. The consequences of climate-driven stop-over sites changes on migration schedules and fitness of Arctic geese. - J. Anim. Ecol. 77: 654-660.
Bauer, S. et al. 2011. Cues and decision rules of animal migration. - In: Milner-Gulland, E. J. et al. (eds), Animal migration - a synthesis. Oxford Univ. Press, pp. 68-87.

Bearhop, S. et al. 2005. Assortative mating as a mechanism for rapid evolution of a migratory divide. - Science 310: 502-504.

Beauchamp, G. 2012. Foraging speed in staging flocks of semipalmated sandpipers: evidence for scramble competition. - Oecologia 169: 975-980.

Bensch, S. et al. 1999. Morphological and molecular variation across a migratory divide in willow warblers, Phylloscopus trochilus. - Evolution 53: 1925-1935.

Bensch, S. et al. 2009. Genetic, morphological, and feather isotope variation of migratory willow warblers show gradual divergence in a ring. - Mol. Ecol. 18: 3087-3096.

Berthold, P. et al. 1992. Rapid microevolution of migratory behavior in a wild bird species. - Nature 360: 668-670.

Blonder, B. et al. 2012. Temporal dynamics and network analysis. - Meth. Ecol. Evol. 3: 958-972.

Boulet, M. and Norris, D. R. 2006. The past and present of migratory connectivity. - Ornithol. Monogr. 61: 1-13.

Bradley, C. A. and Altizer, S. 2005. Parasites hinder monarch butterfly flight: implications for disease spread in migratory hosts. - Ecol. Lett. 8: 290-300.

Brodersen, J. et al. 2008. Ecosystem effects of partial fish migration in lakes. - Oikos 117: 40-46.

Colbeck, G. J. et al. 2013. Groups of related belugas (Delphinapterus leucas) travel together during their seasonal migrations in and around Hudson Bay. - Proc. R. Soc. B 280: 20122552.

Conradt, L. and List, C. 2009. Group decisions in humans and animals: a survey. - Phil. Trans. R. Soc. B 364: 719-742.

Eichhorn, G. et al. 2009. Skipping the Baltic: the emergence of a dichotomy of alternative spring migration strategies in Russian barnacle geese. - J. Anim. Ecol. 78: 63-72.

Fagan, W. F. et al. 2012. Leadership, social learning, and the maintenance (or collapse) of migratory populations. - Theor. Ecol. 5: 253-264.

Fleming, T. 2004. Nectar corridors: migration and the annual cycle of lesser long-nosed bats. - In: Nabhan, G. (ed.), Conserving migratory pollinators and nectar corridors in western North America. Univ. of Arizona Press, pp. 23-42.

Fraser, D. J. and Bernatchez, L. 2005. Adaptive migratory divergence among sympatric brook charr populations. - Evolution 59: 611-624.

Gaidet, N. et al. 2012. Understanding the ecological drivers of avian influenza virus infection in wildfowl: a continental-scale study across Africa. - Proc. R. Soc. B 279: 1131-1141.

Godley, B. J. et al. 2010. Unravelling migratory connectivity in marine turtles using multiple methods. - J. Appl. Ecol. 47: 769-778.

Hall, R. J. et al. 2014. Greater migratory propensity in hosts lowers pathogen transmission and impacts. - J. Anim. Ecol. 83: $1068-1077$.

Harrison, X. A. et al. 2010. Cultural inheritance drives site fidelity and migratory connectivity in a long-distance migrant. - Mol. Ecol. 19: 5484-5496.

Harrison, X. A. et al. 2011. Carry-over effects as drivers of fitness differences in animals. - J. Anim. Ecol. 80: 4-18.

Hedenström, A. et al. 2007. Migration speed and scheduling of annual events by migrating birds in relation to climate change. - Clim. Res. 35: 79-91.

Hein, A. M. et al. 2012. Energetic and biomechanical constraints on animal migration distance. - Ecol. Lett. 15: 104-110.

Holdo, R. M. et al. 2007. Plant productivity and soil nitrogen as a function of grazing, migration and fire in an African savanna. - J. Ecol. 95: 115-128.

Holt, R. D. 2008. Theoretical perspectives on resource pulses. - Ecology 89: 671-681. 
Holt, R. D. and Dobson, A. P. 2006. Extending the principles of community ecology to address the epidemiology of host-pathogen systems. Disease ecology: community structure and pathogen dynamics. Oxford Univ. Press, pp. 6-27.

Hope, D. et al. 2014. Mortality-minimizing sandpipers vary stopover behavior dependent on age and geographic proximity to migrating predators. - Behav. Ecol. Sociobiol. 68: 827-838.

Hoye, B. J. et al. 2011. Reconstructing an annual cycle of interaction: natural infection and antibody dynamics to avian influenza along a migratory flyway. - Oikos 120: 748-755.

Hoye, B. J. et al. 2012. Host behaviour and physiology underpin individual variation in avian influenza virus infection in migratory Bewick's swans. - Proc. R. Soc. B 279: 529-534.

Hudson, P. J. et al. 2002. Ecology of wildlife diseases. - Oxford Univ. Press.

Iwamura, T. et al. 2013. Migratory connectivity magnifies the consequences of habitat loss from sea-level rise for shorebird populations. - Proc. R. Soc. B 280: 20130325.

Johansson, J. et al. 2015. The eco-evolutionary consequences of interspecific phenological asynchrony - a theoretical perspective. - Oikos 124: 102-112.

Jorgensen, S. J. et al. 2010. Philopatry and migration of Pacific white sharks. - Proc. R. Soc. B 277: 679-688.

Kamiya, T. et al. 2014. What determines species richness of parasitic organisms? A meta-analysis across animal, plant and fungal hosts. - Biol. Rev. 89: 123-134.

Keeling, M. J. and Rohani, P. 2008. Modeling infectious diseases in humans and animals. - Princeton Univ. Press.

Kölzsch, A. et al. 2015. Forecasting spring from afar? Timing of migration and predictability of phenology along different migration routes of an avian herbivore. - J. Anim. Ecol. 84: 272-283.

Marra, P. P. et al. 2010. Migratory connectivity. - In: Breed, M. D. and Moore, J. (eds), Encyclopedia of animal behavior. Academic Press, Oxford Press.

McNamara, J. M. et al. 2011. Cues and the optimal timing of activities under environmental changes. - Ecol. Lett. 14: 1183-1190.

Middleton, A. D. et al. 2013. Animal migration amid shifting patterns of phenology and predation: lessons from a Yellowstone elk herd. - Ecology 94: 1245-1256.

Miller, N. G. et al. 2012. Migratory connectivity of the monarch butterfly (Danaus plexippus): patterns of spring re-colonization in eastern North America. - PLoS ONE 7: e31891.

Møller, A. P. and Szep, T. 2011. The role of parasites in ecology and evolution of migration and migratory connectivity. - J. Ornithol. 152: 141-150.

Mooij, J. H. et al. 1999. White-fronted goose Anser albifrons albifrons. - In: Madsen, J. et al. (eds), Goose populations of the Western Palearctic. A review of status and distribution. Wetlands Int. and Natl Environ. Res. Inst., pp. 94-128.

Supplementary material (available online as Appendix oik-02706 at <www.oikosjournal.org/appendix/oik-02706>). Appendix 1.
Morrissette, M. et al. 2010. Climate, trophic interactions, density dependence and carry-over effects on the population productivity of a migratory Arctic herbivorous bird. - Oikos 119: 1181-1191.

Moussy, C. et al. 2013. Migration and dispersal patterns of bats and their influence on genetic structure. - Mamm. Rev. 43: 183-195.

Nakazawa, T. and Doi, H. 2012. A perspective on match/mismatch of phenology in community contexts. - Oikos 121: 489-495.

Norris, D. R. and Marra, P. P. 2007. Seasonal interactions, habitat quality, and population dynamics in migratory birds. - Condor 109: 535-547.

Orell, P. et al. 2007. Synchrony in the downstream migration of smolts and upstream migration of adult Atlantic salmon in the subarctic River Utsjoki. - J. Fish Biol. 71: 1735-1750.

Reed, T. E. et al. 2010. Phenotypic plasticity and population viability: the importance of environmental predictability. - Proc. R. Soc. B 277: 3391-3400.

Reperant, L. A. et al. 2010. Spatial and temporal association of outbreaks of $\mathrm{H} 5 \mathrm{~N} 1$ influenza virus infection in wild birds with the $0 \mathrm{C}$ isotherm. - PLoS Pathog. 6: e1000854.

Saino, N. et al. 2010. Sex-related variation in migration phenology in relation to sexual dimorphism: a test of competing hypotheses for the evolution of protandry. - J. Evol. Biol. 23: 2054-2065.

Salomonsen, F. 1955. The evolutionary significance of bird migration. - Biol. Medd. 22: 1-62.

Seewagen, C. L. et al. 2013. Stopover refueling rate underlies protandry and seasonal variation in migration timing of songbirds. - Behav. Ecol. 24: 634-642.

Skoglund, H. et al. 2011. Competitive interactions shape offspring performance in relation to seasonal timing of emergence in Atlantic salmon. - J. Anim. Ecol. 80: 365-374.

Stahl, J. et al. 2006. Subtle interplay of competition and facilitation among small herbivores in coastal grasslands. - Funct. Ecol. 20: 908-915.

Taylor, C. M. and Norris, D. R. 2010. Population dynamics in migratory networks. - Theor. Ecol. 3: 65-73.

Webster, M. S. and Marra, P. P. 2005. The importance of understanding migratory connectivity. - In: Greenberg, R. and Marra, P. P. (eds), Birds of two Worlds: the ecology and evolution of temperate - tropical migration systems. Johns Hopkins Univ. Press, pp. 199-209.

Webster, M. S. et al. 2002. Links between worlds: unraveling migratory connectivity. - Trends Ecol. Evol. 17: 76-83.

Wittwer, T. et al. 2015. Long-term population dynamics of a migrant bird suggests interaction of climate change and competition with resident species. - Oikos 124: 1151-1159.

Yang, L. H. and Rudolf, V. H. W. 2010. Phenology, ontogeny and the effects of climate change on the timing of species interactions. - Ecol. Lett. 13: 1-10. 